\title{
Inter-university Project Based on LEGO NXT
}

\author{
Adam Pilat ${ }^{1}$, Andrew J. Kornecki ${ }^{2}$, Jean-Marc Thiriet ${ }^{3}$, Wojciech Grega ${ }^{1}$, Ondrej Rysavy ${ }^{4}$
}

\begin{abstract}
The paper describes the mechanism for involving students from multilingual, geographically separated institutions in a coordinated educational experience and presents the observations and evaluation of outcomes related to the collaborative project. The project was realized as a part of ILERT (International Learning Environment for Real-Time Software Intensive Control System) EU/US activity sponsored by FIPSE/EC Atlantis Program. As a part of the pilot implementation phase of the ILERT project, international students teams have worked on robotic design and control experiments with LEGO MINDSTORMS NXT kits. The project has engaged on-site students and faculty of universities from four countries (USA, Poland, France, and Czech Republic). The robot maze navigation problem has been defined as an example. LEGO-MINDSTROMS NXT kit has been selected as a common platform for students teams. A Web-based Project Management System (WebPMS) has been used to improve communication and test Internet based tools for inter-university collaboration. Final evaluation of the project outcomes, including students survey results, are presented in this paper.
\end{abstract}

\section{INTRODUCTION}

$\mathrm{I}_{\mathrm{F}}^{\mathrm{L}}$ LERT project stands for International Learning Environment for Real-Time Software Intensive Control Systems. Considering demand for efficient development of quality Real-Time Software-Intensive Control (RSIC) systems, ILERT presents methodology for creation of multinational, interdisciplinary curriculum producing graduates capable of working efficiently in teams engaged in international collaboration on safety-related RSIC systems [6]. ILERT engages four university partners proposing a pilot study leading to establishing international curriculum framework focusing on the important aspect of the computer/system/control/software engineering education. The framework was designed to be a model for other engineering programs across the Atlantic. Embry Riddle

Manuscript received January 15, 2009. This work was supported by the U.S. Department of Education under FIPSE Atlantis Program Grant P116J06000 and European Commission Grant 2006-4563/006-001 CPTCPTUSA, and from the regular budgets of EU partner universities.

Adam Piłat is with the AGH University of Science \& Technology,

Poland, (phone: +48 12 6172094; fax: +48 12 6341568; e-mail: ap@ ia.agh.edu.pl)

Andrew J. Kornecki is with the Embry-Riddle Aeronautical University, USA, (e-mail: kornecka@erau.edu).

Jean-Marc Thiriet is with the Grenoble Université, France, (e-mail: jeanmarc.thiriet@ujf-grenoble.fr).

Wojciech Grega is with the AGH University of Science \& Technology, Poland, (e-mail: wgr@agh.edu.pl).

Ondrej Rysavy is with the Brno University of Technology, Czech

Republic, (e-mail: rysavy@fit.vutbr.cz).
Aeronautical University (Daytona Beach, FL, USA), AGH University of Science and Technology (Krakow, Poland), University of Technology (Brno, Czech Republic), and Laboratoire d'Automatique (Grenoble, France) recognized the demand for efficient development of quality RSIC systems and need for an International Learning Environment.

Among several tasks realized in the ILERT project, one was dedicated to the inter-university cooperation in the students' laboratory activity.

The current trend in engineering curricula applies the concept of "learning by experiments" or "learning by projects" [1]. The approach gives the students the opportunity to get familiar with practical problems that the organizations and the development teams may run into in real situations. It was found, that learning achievements resulting from the application of a collaborative work methodology based on such approach are superior [3].

Such "learning by doing" concept was also proposed for the collaborative project as a part of the pilot ILERT implementation phase. During the research phase of the ILERT, an intensive literature study demonstrated that several universities have worked with robotic design and control experiments using LEGO MINDSTORMS kits [2], [11]. The LEGO kits give the students a flexible and userfriendly platform, supporting a variety of robotics projects. The published hardware [7] and software [8] documentation makes the LEGO ${ }^{\circledR}$ MINDSTORMS $®$ NXT system open for modifications and new applications. Several authors are replacing the conventional operating system of the MINDSTORMS microcontroller, supporting kids-friendly graphic programming paradigm, with a real-time operating system suitable for programming in $\mathrm{C} / \mathrm{C}++$ or Java. Most popular is NXC language [5], which supports internal ARM7 microcontroller commands. This upgrade might be advantageous for more realistic real-time control applications. The "upgraded" LEGO MINDSTORMS experiments can teach students the importance of real-time computing, periodic tasks development, concurrency, timers, embedded processor size, and cost versus performance trade-offs within the context of control systems. 


\section{Collaborative Project}

\section{A. The Project Objectives}

demand as much as a good preparation in math. The technical objective of the project was to develop software packages for two robots that cooperate in searching for a target in a simple maze. The use of rigorous software development process was emphasized and the comprehensive documentation was expected as an integral part of the project's outcomes. Team cooperation and related intensive inter- and intra-team communication was also assumed. Each team was responsible for their developed components from the initial phases of analysis and design through component implementation to integration and testing.

The Project Learning Objectives were twofold:

o Effective work in multicultural multidisciplinary team:

- Team organization and planning

- Team communication

- Project management

o Development of an RSIC system:

- Developing real-time embedded software

- Hardware/Software system integration

- Implementation of digital control in real time

- Design/implement communication protocols

\section{B. Why LEGO MINDSTORMS NXT platform?}

In a preparation for the project Partners had considered several issues like diverse background of the teams, need for focusing on the software/control/communication aspects of the technical project, as opposed to mechanical/robotics ones. We were thus looking for common hardware and software platform ready to use and one which would have a number of features. The selected LEGOMINSTROMS NXT platform:

- allows to create Multidisciplinary (and Interdisciplinary) projects

- can be applied in many fields of engineering: automatic, robotics, mechanics, electronics, informatics

- $\quad$ has open architecture for hardware and software tasks

- allows to demonstrate both theory aspects and practical control algorithms, communication and data exchange problems, remote control and communication (like position control, speed control, path tracking, etc.)

- can be adapted for team cooperation, project organization both local and global, work plans, schedule, timetables, international meeting and competitions.

\section{Embedded Systems Programming}

One of the most important lessons for the control engineering students is the implementation of theory in practical applications, specifically when using micro-
The results of the industry survey (analysis of the industry requirements on graduates in the RSIC domain, [9]) suggest that cooperation in multidisciplinary teams is very much in controller based systems. The limitations of hardware resources causes that some algorithms implementation is not feasible and a few variants must be studied before implementation.

Another important issue is teaching low-level programming when using hardware [4]. Such approach enhances didactical value of the activity due to the possibility of observation and analysis of the hardware behavior. The programmer should have a full control over the hardware and be able to assess the performance of the developed code.

The programming solutions related to LEGO NXT are represented by NXC, a free and open source. The NXC (Not Exactly $C$ ) is a text-based language for MINDSTORMS $\circledR$ NXT robot microcontroller programming with multitasking features, based on standard LEGO firmware [8]. With free Integrated Development Environment (IDE) [5] it is possible to create quite complex and advanced data acquisition and control algorithms operating concurrently and in real-time, including file management and Bluetooth communication features. Using text-based language, the programming skills of students, their understanding of data precision, time dependencies, and I/O devices access methods can be greatly improved.

Sample programs demonstrating the multitasking feature, data login to memory and files, robot movement control, sensors measurement, and motor controller tuning were prepared in advance and used at the initial project stage (See: http://www.ilert.agh.edu.pl/ "Collaborative Project" link ).

\section{Project Objectives}

The system to be build and studied included two LEGO robots and a base station on a general purpose workstation with enabled Bluetooth communication. Bluetooth dongle shipped with LEGO MINDSTORMS NXT kit was preferred, as some problems have been experienced with various Bluetooth devices.

Robot building instruction prepared using LEGO Draw Designer Software (see Fig. 1) and available on the project website, has constituted a common base for all project teams (See: http://www.ilert.agh.edu.pl/ "Collaborative Project" link). 


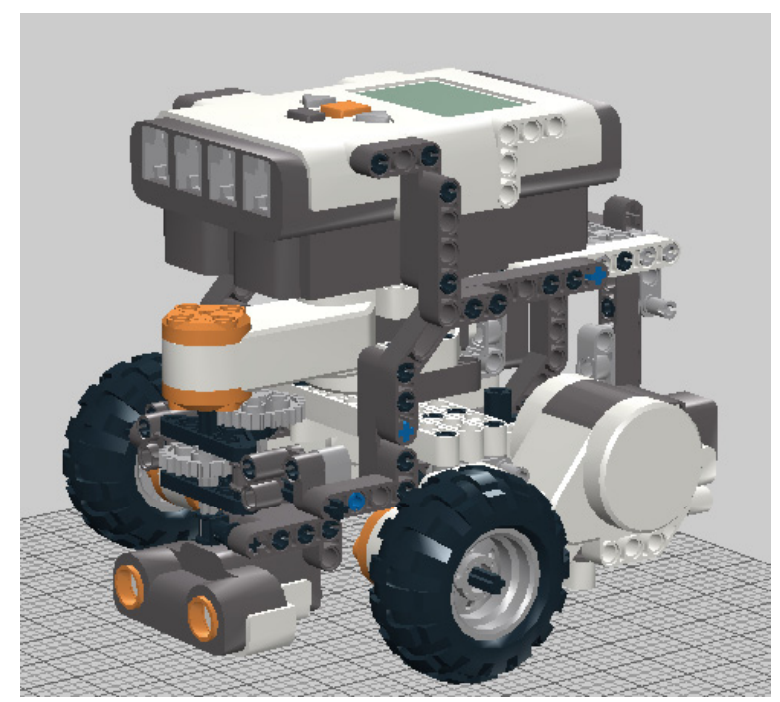

Fig. 1. Design of three wheels robot construction

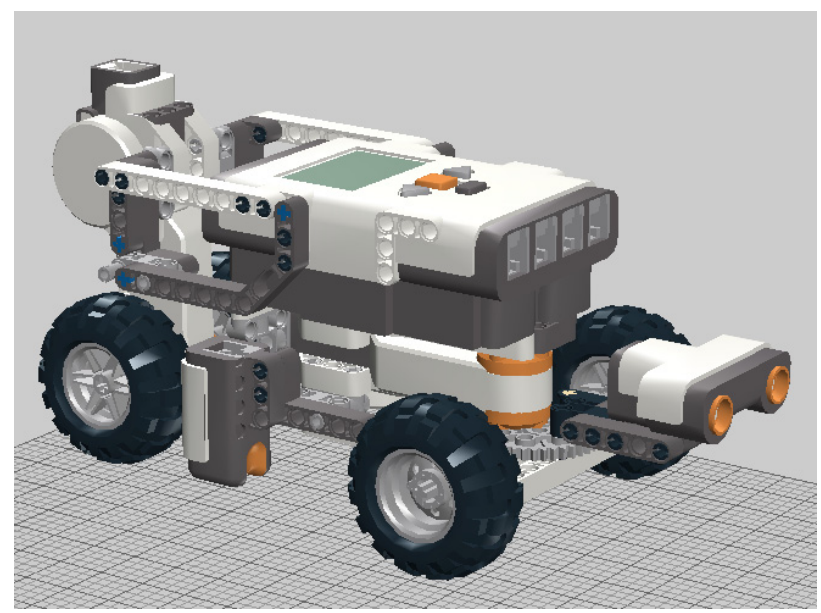

Fig. 2. Design of four wheels robot construction

The two robots were considered to work only within the operating area $1.5 \mathrm{~m} \times 2.0 \mathrm{~m}$ (Fig.3). There are five gray squares printed on the Styrofoam board. The gray squares in each corner are designed to provide the robots the position information. The squares are of different shades of gray: $15 \%, 30 \%, 45 \%$ and $60 \%$. The square in the center must be $80 \%$ or more to be recognized as the target position.

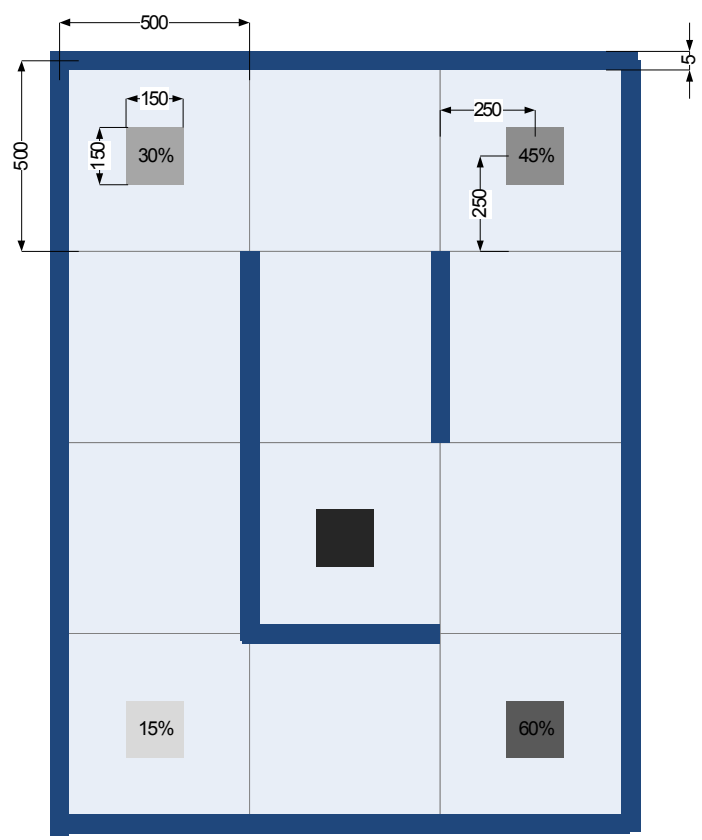

Fig. 3. Operating Scenario

The robots were assigned different roles (Fig.4):

- The Leader Robot starts in one of the four corners and, according the implemented algorithm, it is searching for the black square that is positioned in the center of the maze.

- The Follower Robot starts in one of the four corners (which may be different that the starting corner of the Leader Robot) and according the information received from the Leader robot it attempts to find the black corner is a shortest possible time.

The leader robot can use the available sensors in its way through the maze. No external method for navigating the robot to the target should be used.

The problem of target search in the maze was conceived as a practical example ("a rescue problem"). The basic problem is accurate and precise motor control. The error in the motor control can affect the correct navigation and robot movements. From the didactical aspects of the project, it is not so important that the robot moves, but how precise it operates and what is the accuracy of the controlled devices.

A host workstation i.e. a personal computer with BrixCC LEGO programming environment was required. A custom program needs to be designed and implemented so the robot(s) can communicate with the host and thus their movements can be monitored and displayed. The host monitoring program is an added component and can be created using any off-the-shelf package. The Bluetooth connection must be active, with robot visibility set, the robot names and ports configured. Host workstation must have an active internal or dongle Bluetooth connection. 


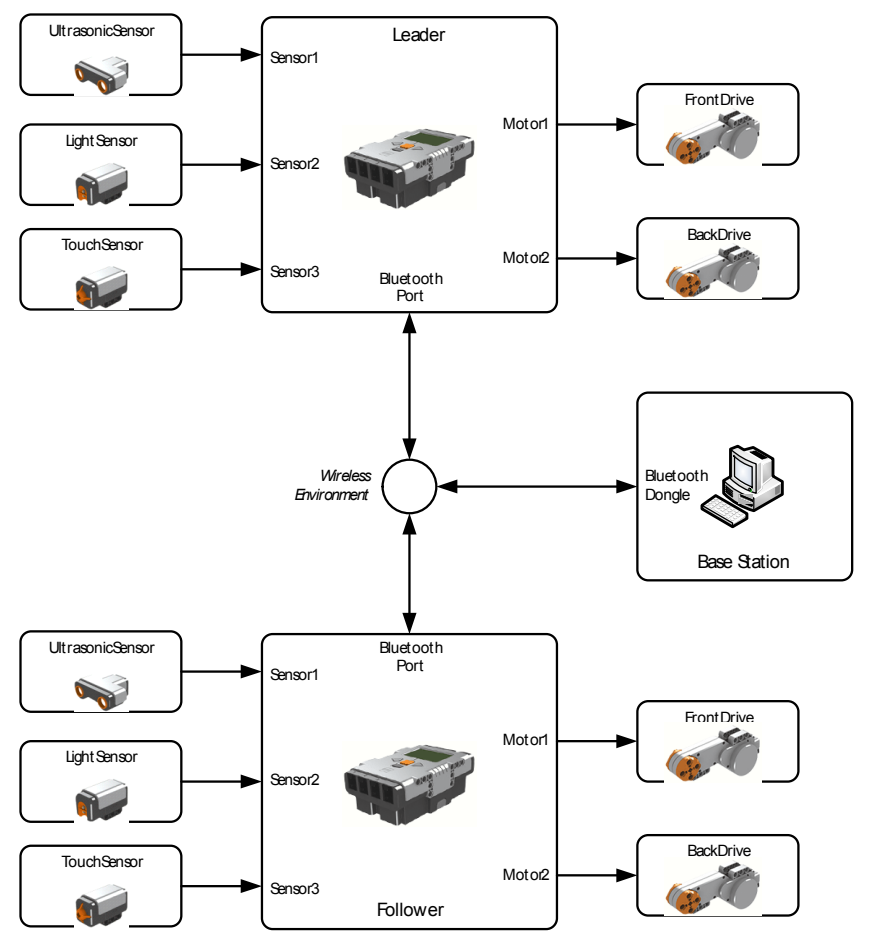

Fig. 4. Overall system architecture

\section{E. Project Management}

Due to composition of ILERT consortium proposing a pilot project based on inter-university collaboration, the LEGO project had four instructors/mentors affiliated with the four national teams assigned to the laboratories at home universities.

Communication is the most important component within any project. The success of most projects depends upon communication skills and techniques.

The following media and channels have been selected to establish communication between the project members:

- dedicated to Collaborative Project link at the ILERT project website (www.ilert.agh.edu .pl),

- forum operating at WebPMS,

- task monitoring, logs and e-mailing at WebPMS,

- files storage,

- regular e-mail.

All start-up information and final artifacts were placed and linked from the Collaborative Project Web page. The project assumptions, robot building instructions and sample programs were initially uploaded. Final project reports are also presented there.

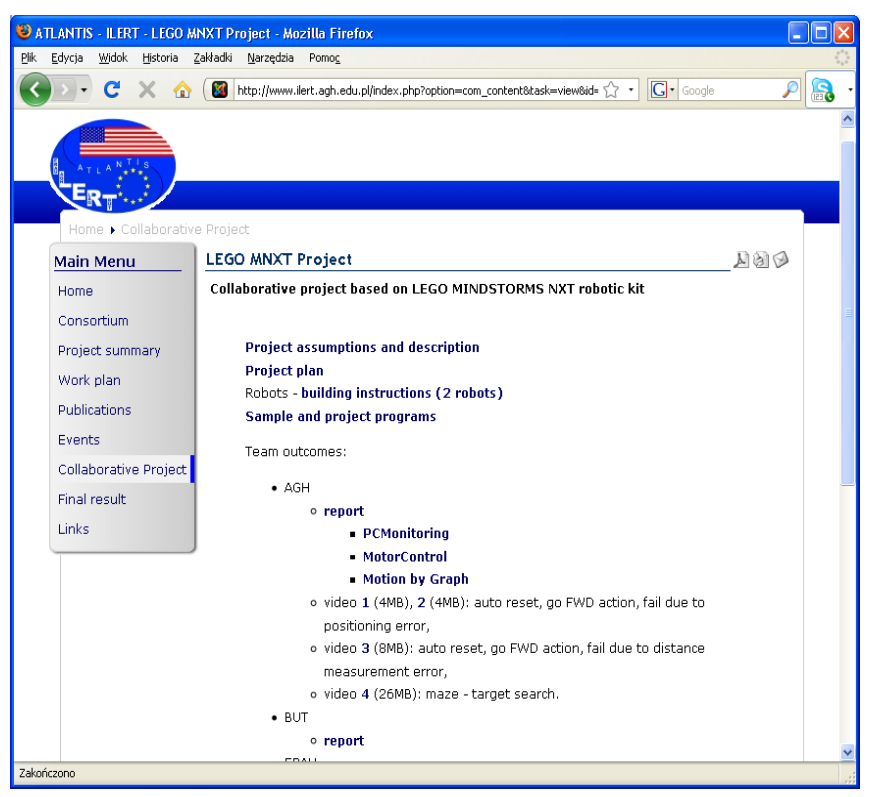

Fig. 5. Collaborative Project Web page

The project management system (WebPMS) based on the dotProject open software platform [10] has been introduced to support work organization and the information exchange (Fig.6). This system was tested before the project start-up for the project members to register and login. Also the course instructors had appropriate rights for project modification via insertion of a new tasks, forums and topics. The overall structure of forum and tasks has been initialized.

The project members were using Task Logs and entering work time. We needed to use this mechanism for all project members to monitor activity and workload. The registration of work progress (including time) is critical. It allows observing the learning process for particular student, to analyze task effort, estimate project time and costs.

Figure 6 presents an example of the Task Logs system. One can notice that there are some entries and register working hours. The e-mailing feature was used to inform project participants about information entered. This WebPMS feature was useful and it is recommended for other projects because it works as a reminder.

The discussion about project problems took place at the forum being a part of the WebPMS. With our project we tried to implement a not moderated forum. However, in a few cases there was a strong request to make the final decision by the course instructor or the mentor. 


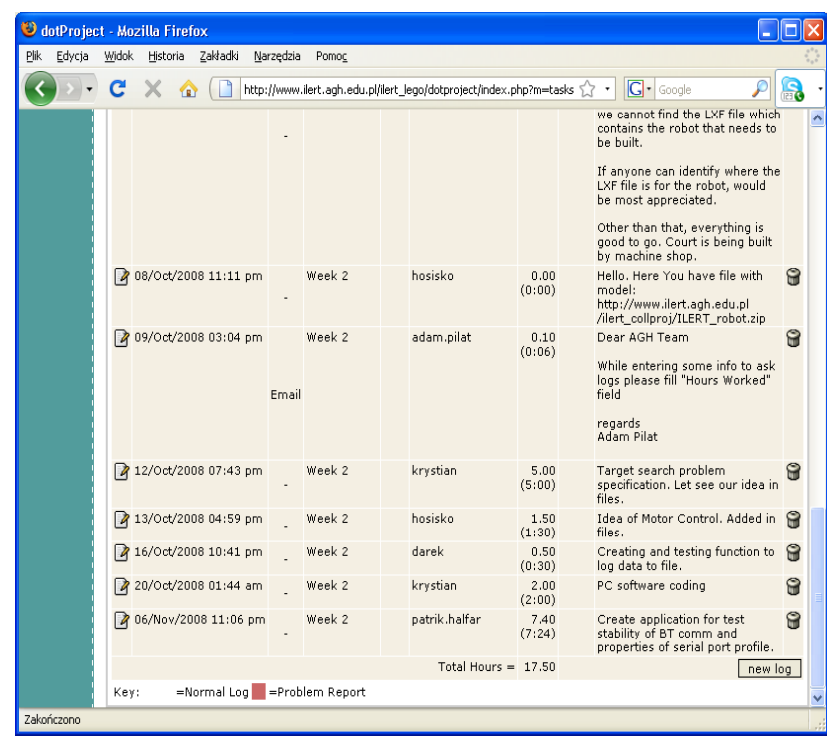

Fig. 6. WebPMS - Task Log window

Using WebPMS the Files Folder was opened for project members' access. The documents, programs and presentations were placed in the common area at the web server for easy access and exchange between Partner Universities.

\section{STUDENTS SURVEY}

The students participating in the collaborative project were asked to fill out a prepared survey. The questions were grouped in a few topics: preparation stage, local team work and infrastructure, cooperation, software, new skills (see "Final Results" link at the ILERT web page).

\section{Project Evaluation AND OUtComes}

This experimental activity reflected the concept of a project in industrial environment. As a matter of project design, the tasks were not precisely defined and the students had opportunity to explore variety of solutions and tradeoffs. They were free to define both the product to be created and the process to accomplish the realization of the product. Such situation is very different from conventional laboratory exercises, since the project was new and not a replication of one done before. The main objective was to observe the teamwork in the interdisciplinary and international setting.

The project has not been a complete success. However, most of the intended objectives have been accomplished. Accomplishments are as follows:

- Mutual collaboration: testing of variety of communication methods, exchange of ideas and comments over Internet

- Realization of tasks assigned to the teams

- Completion of the robot control software

- Experiments with the real robot

- Completion of the project documentation

- Reports and presentations by the teams
There was misunderstanding with the assignment of a single person responsible for overall project supervision (manager/leader). Due to the international inter-university collaboration, the project had four instructors/mentors affiliated with the national teams. The project plan identified a rotating leadership with one team instructor responsible for the lead in a specific week. The leader needed to be fully familiar with the project objectives and be able to evaluate feasibility of achieving these objectives. The responsibility of such person was monitoring the internet discussion forum and reacting in case of situation requiring attention. An example of such situation was when one of the teams discovered that trigonometric calculation designed to determine robot position were not feasible. Instead, a new concept was proposed in a form of discretization of the area and monitoring the movement by moving from square to square. However, the teams did not react to the new concept and for nearly two weeks there was no agreement on how to proceed. Another example was during discussion regarding the robot control and the level of precision due to gear and ultrasonic sensor inaccuracies.

The teams failed to record rigorously their work efforts. This is the reason why the complete record of the overall team project effort was missing. It was assumed that the dotProject software, which allows for rigorous recording of time and task comments, will be used by all participants and thus allow us for a post-mortem assessment of the project. Unfortunately, despite repeated appeals and encouragement, the students failed to log their time in all instances of the project.

Related to lack of the overall project lead - there was also misunderstanding who was the designated forum moderator. As specified above, the moderator should be the course instructor identified as the lead in the specific project week.

Timely delivery of the assigned artifacts and reporting was lacking. This included also timely launching of the tasks for the given week and timely reporting of the outcomes. Lack of a rigorous reporting scheme was demobilizing factor and impediment to the progress of the project. However, the report framework was prepared and all teams used it successfully, but with no consistency on weekly basis. It is imperative that rigorous weekly reporting and sharing information with all teams is critical for the project success. It was found at the final stage of the project that the defined tasks were too complex and were not decomposed properly into smaller subtasks. Some of the students left the project decreasing the team size (ERAU from 3 to 2, AGH from 4 to 3 , BUT from 3 to 1 , LAG from 3 to 2). However, the tasks were not revised in time and re-planning did not take place.

The electronic forum was helpful in some problems solving, although not. All participants were active. Due to the absence of the general project leader some problems was not solved definitely. The files folder was a useful element where the project outcomes such as team presentation, program files, documents, report and surveys were stored. The task logs were also a valuable part of the system to show the project time line and task details. The task reports 
and logs were showing the team work progress and allowed to send via e-mail messages to the project members to help solve problems or just to inform them accordingly. As observed, not every event has been logged into the WebPMS systems. Notoriously, the students kept forgetting to fill the task logs despite them being asked to do it several times.

Summarizing the students' feedback one can observe that the initial stage was prepared quite well although documentation should be much more precise and some project aspects described better. The application of WebPMS system has been considered a very good idea but unfortunately the tool was not used regularly.

Local teams were operating smoothly with well organized local communication and meetings. However, the international cooperation was much weaker due to marginal information exchange. One of these reasons is that the project manager and the forum moderator roles were not adequately assigned. In some cases the discussion was suspended for a long time, or some questions remained without answers.

The project workload cannot be estimated more precisely due to the missing information at WebPMS systems student did not log their work progress regularly, so it is impossible to calculate the amount of time they contributed to the project. As estimated and declared the number of working hours was: 40-AGH, 50-BUT, 25-ERAU and 45BUT

Some students gave us additional free comments: "Most of hourly-costs were used for planning and fighting with errors on Lego's brick's. “A lot of time was lost because of project inaccuracies." "Project was very interesting but with more communication it would be much better."

\section{V.CONCLUSIONS}

The presented project was experimental in the sense of having single project undertaken by teams representing four schools located in geographically distant countries with all collaboration over Internet only. The used LEGO platform proved flexible enough to achieve the technical objectives.

For improvement in the area of the process it is imperative to work out appropriate mechanisms for data transfer and confirmation when receiving the information between the teams. The suggested procedure is to include in weekly report the information regarding e-mail exchanges, forum discussions, and tasks deliverables. Assignment of a single person responsible for overall project supervision (manager/leader) is important. One of his duties can be monitoring how information flows between project teams.

Rigorous logging of the work effort is critical. The best if it would be done fully automatic, which is easy when the work is done in front of computer. Appropriate planning and monitoring the effort is critical for any process-driven engineering organization. The project objective is not only to deliver the product but also to teach the teamwork, work organization, and following a defined process. Estimation of overall project effort based on the collected data was not possible due to missing information from students working on the project.

To succeed with the project management and issue tracking the evaluation of systematic and correct entries should be a part of the final student grade. This will perhaps motivate the students better to submit the required information. The project shortcomings confirmed that teaching project and time management ought to be realized as a part of engineering curricula.

\section{REFERENCES}

[1] Bassam A. Hussein, Kjetil Nyseth, "A method for learning in project management, 'Learning by projects' “, 9th International Workshop on Experimental Interactive Learning in Industrial Management, "New Approaches on Learning, Studying and Teaching", Espoo, Finland, June 5-7, 2005

[2] Gawthrop P. J., McGookin E., "A LEGO®-Based Control Experiment”, IEEE Control Systems Magazine, v.24, October 2004, pp. 43-56

[3] Grega W., Piłat A., "Platforms for Laboratory Experiments: Low-cost Kits vs. Dedicated Trainer"s, Proceedings of 18th EAEEIE Conference, Praha July 2-4, ISBN 978-80-01-03745-4

[4] Grega W., Pilat A., (2008), "Real-time Control Teaching Using LEGO ${ }^{\circledR}$ MINDSTORMS ${ }^{\circledR}$ NXT Robot", Proceedings of RTS'08 International Conference on Computer Science and Information Technology, Wisla, Poland (ISSN 1896-7094, CD-Edition)

[5] Hansen J., "Not eXactly C (NXC) Programmer's Guide", 2007

[6] Kornecki, A., Hilburn, T., Grega, W., Thiriet, J-M.Sveda, M. (2008), "ILERT - International Learning Environment for Real-Time Software-Intensive Control Systems" accepted for publication JAMRIS - Journal of Automation, Mobile Robotics and Intelligent Systems, Vol. 1, 2009

[7] LEGO ${ }^{\circledR}$ MINDSTORMS ${ }^{\circledR}$ Hardware Developer Kit (HDK), http://mindstorms.lego.com, LEGO Group, 2006

[8] LEGO ${ }^{\circledR}$ MINDSTORMS ${ }^{\circledR}$ Software Developer Kit (SDK), http://mindstorms.lego.com, LEGO Group 2006

[9] Pilat, A., Kornecki, A., Thiriet, J-M., Grega, W., Sveda, M., (2008), "Industry Feedback on Skills and Knowledge in Real-Time Software Engineering", 19th EAEEIE Annual Conference, Tallinn, Estonia, June 29 - July 2, 2008 http://eaeeie.ttu.ee/, ISBN:978-1-4244-2009-4

[10] Project Management System, http://www.dotProject.net

[11] Wang E. L., LaCombe J., and Rogers C., "Using LEGO® Bricks to Conduct Engineering Experiments," Proceedings of the ASEE Annual Conference and Exhibition, session 2756, 2004 